\title{
Clinical outcome and prognostic factors for central neurocytoma, a study of 14 cases
}




\title{
Clinical outcome and prognostic factors for central neurocytoma, a study of 14 cases
}

\author{
Mohamed Abdel Bari Mattar, Ashraf El Badry \\ Department of Neurosurgery, Mansoura University, EGYPT
}

\begin{abstract}
Background: Since they're rare, the intraventricular neoplasms "central neurocytoma" best management got diverse, and mystifying. Aim of the work: to assess outcome for patients with central neurocytoma and value of specific factors like tumor size, surgical resection extent, atypia, and concomitant other modality of treatments by radiotherapy in their survival. Patients \& methods: 14 patients ( 8 males, 6 females) were managed surgically between 2012 and 2016. They were assessed clinically, radiologically and their outcome in relation to selected factors. Results: Median age at diagnosis was 28.3 years in average (range 16-58). Median follow-up was 32.2 months.we lost 2 cases to follow-up in post-operative period. Six patients had recurrent of neurocytoma tumours.Five patients (35.7\%) obtained gross total resections (GTR) while 9 patients (64.2\%) had subtotal resections (STR). Two STR patients (14.2\%) received dose of radiation post operatively that significantly enhanced overall PFS $(\mathrm{p}=0.047)$. our series data propose that radiation therapy following (STR) usually increase (PFS). Two patients proved to have atypical neurocytoma by pathological reports died at 2.3 and 10.2 months after the microsurgical procedures. MIB- 1 tagging index higher than $4 \%$ is an indicator for poor outcome. We used Kaplan-Meier beside Cox proportional hazards methods in determining the Progression-free survival (PFS) in our study. Conclusion: the extent of surgical resection may improve the neurological condition but not the survival, Atypia was the most important factor determine the recurrence \& survival while radiotherapy improve the survival quietly.
\end{abstract}

Key words: Atypical Central neurocytomas, Benign central nervous system tumor, Central neurocytomas, MIB-1 tagging index, Progression free survival

\section{Introduction}

Central neurocytoma $(\mathrm{CN})$ is an uncommon central nervous system tumor which encompass only $0.1-0.5 \%$ of all primary brain tumors. It was firstly described by Hassoun and colleagues in 1982 and, by the early 1990s; it had become a well-defined clinical and pathological entity [8]. It was classified by the World Health Organization in 
the year of 2000 A.D. as grade II but malignant variant have been reported [13]. However only not more than one thousand patients with $\mathrm{CN}$ have been stated worldwide [10]. Earlier studies showed that $\mathrm{CN}$ derived from ectodermal/nervous system tissue and mainly located in deep mid-line structures near the foramen of Monro (so it can arise from the walls of the lateral ventricles, fornix, the septum pellucidum), it is typically situated supratentorially filling the lateral ventricle(s) and/or the third ventricle with evolving of neurological symptoms related to intracranial hypertension (headaches beside plus or minus visual disturbances. The clinical symptoms period usually not more than 6 months [25]. Neurocytomas may be found away from the ventricles and have been defined by WHO as an extraventricular neurocytomas (EVNs). This extraventricular neurocytomas (EVNs) beside its different places, it have a broader scope in morphology compared with intraventricular neurocytomas. [13] $\mathrm{CN}$ frequently arose in youth period, $70 \%$ of $\mathrm{CN}$ patients present in the third and fourth decade of life. Tumor incidence shows no significant gender preponderance $[3,15,24]$. In spite of most $\mathrm{CN}$ were benign and most of the patients with $\mathrm{CN}$ survived for long time after a gross total removal but atypical pathological variation have been described and even residual tumors parts after surgery may transformed to have possibility of more malignant features [11, 23]. Surgery is the basic traditional treatment for these cases and the degree of surgical excision is the major element affecting the clinical outcome. Furthermore, radiotherapy (RT) can efficiently control remaining tumor following partial microsurgical removal or in recurrent cases [12, 17]. The standard guidelines as a stepladder treatment for this neoplasm has not so far been recognized, particularly that the role of Radiation Therapy after the surgery stays debatable $[7,19,22]$. Central neurocytomas that possess atypical pathologic characteristics act more violently and might be associated with high recurrence rate. The usage of MIB-1 with specific threshold as an objective marker to signifying atypia is debatable $[4,9,21]$. MIB-1 marker have been used in many studies for grading of astrocytoma cases. These studies postulate that E3 ubiquitin-protein ligase MIB1 act as an enzyme which encoded by the human MIB1 gene in regulating apoptosis. [16] The clinical management of neurocytoma remain unclear, In this paper, we discussed the management of these cases and investigated whether initial tumor size, degree of tumor excision, MIB-1 tagging index and postoperative dose of radiotherapy have impact on clinical outcomes or not.

\section{Patients and methods}

All CN patients were treated at Mansoura University hospitals (MUH) between 2011 and 2016. The patients of our study have the following inclusion criteria: (1) primary surgery was achieved at MUH, (2) original management documents was available, and (3) the tumor pathology results were obtainable in the patient's records. All existing cases were included in our study regardless the follow-up period because of the rarity of this tumor, the medical reports were reviewed and the demographic, treatment options used and outcome data were collected. Tumor size was 
calculated by using $\mathrm{T} 1$ imagines post-contrast and T2-weighted brain MRI films in the 3 orthogonal directions, for the tumor volume determination. Degree of tumours excision was clarified according to the operative notes reviewing and post-operative radiology. Gross total resection (GTR) was defined by images as complete tumor surgical removal and neartotal excision while all other surgical techniques as well as partial resection, biopsy with or without cyst aspiration, took in account as subtotal resection (STR). Patients were followed in post-operative period clinically and radiologically (MRI and/or CT) during the post-surgical follow-up period. Tumor evolution was expressed as $\geq 25 \%$ expansion of the above mentioned radiologic tumor volume estimation. Outcomes of interest included progression free survival (PFS) from the date of primary surgery besides overall survival (OS) of the same period.

\section{Statistical analysis}

The evaluation of (PFS) and (OS) was done by using the Kaplan-Meier method. While the log-rank test and univariate Cox proportional hazards methods were applied to validate the following items as prognostic factors, for instance: degree of tumor removal, tumor volume, MIB-1 tagging index, and concomitant postoperative radiotherapy through its affection on PFS and OS. We used $\mathrm{p} \leq 0.05$ as Statistical significance threshold in our research.

\section{Results}

Demographic data: A sum of $14 \mathrm{CN}$ patients (It showed histological and immunohistochemical classical features) were treated in our department but only 12 patients were alive at time of study (Table 1).

Two patients were lost to follow-up afterward primary surgery but were still incorporated in the study. The proportion of male to female was 1.3:1. The median age at tumor diagnosis was 28.3 years (range 16.258.4 years). All patient suffered from manifestation of high ICP and three cases had seizures. Radiological results: CT brain of these cases showed a well demarcated, iso- or faintly hyper dense in the supra tentorial intraventricular space. Calcifications have been seen in eight cases. Images obtained after administration iodide contrast substances displayed moderately homogeneous enhancement lesions in the images of all cases. While Cystic changes appeared in 6 cases.

MRI images of the cases revealed: large heterogeneously enhancing intraventricular mass which has a "soap bubble" appearance on T2. In all cases and broad attachment to the septum pellucidum. Most patients (12 cases) had their tumors in the cavity of the lateral ventricle, and (2 cases represent 14\%) experienced midline ventricular lesions.

The median biggest tumor dimension was $6.7 \mathrm{~cm}$ (vary between $3.8-9.6 \mathrm{~cm}$ ) on preoperative MRI examination. Anterior Trans callosal approach (TCA) was selected in six cases, and anterior transcortical approach (TCO) was selected in eight cases. Five of the fourteen cases had GTR, on other hand $64 \%$ of CN patients (9/14) had STR. Massive intraoperative bleeding is crucial point of resectability. All tumors were considered as WHO Grade II on histopathologic examination. Three of the 14 tumors (21\%) were distinguished as atypical on neuropathological study. 
Almost $65 \%(9 / 14)$ of patients had hydrocephalus that mandated the application of a ventriculoperitoneal shunt at different perioperative durations. One patient who had a GTR was given a dose of External Beam Radiotherapy (EBRT) while only two cases with STR (14.2\%) were given radiation therapy after primary surgical excision. The remaining 7 cases did not receive any radiation.

\section{Outcomes}

Follow-up of the study ranged 10.6-43.8 months (Median duration was 32.2 months).it was found that along the study time period, six patients (42\%) developed recurrent or progressive disease while the residual cases of the study did not. Two cases of mortality in the study (14.2\%) were reported. The 2-year PFS was $71 \%$ in our research beside predictable 5years PFS was $52 \%$ (Table 2). The age and gender notably did not influence PFS. As regard tumor size, $\mathrm{p}$ value was 0.79 which statistically significant: PFS for 2-years was 72 $\%$ when tumors size lower or equal to 6.1 while it was $76 \%$ for tumors size higher than $6.1 \mathrm{~cm}$ concerning tumor atypia in pathological examination \& immunohistochemical staining, 2-year PFS was $89 \%$ for MIB tagging higher or equal to 4 versus $49 \%$ for MIB tagging below $4 \%(\mathrm{p}=0.0025)$. The use of radiotherapy following surgery for patients with subtotal resection STR showed that the overall PFS progress was significant with $\mathrm{p}=$ 0.047 . As regard the two mortality cases, one happened 2.3 months after surgery due to intracranial hemorrhage. While the second occurred 10.2 months due to disease progression. Notably both patients had high tumor atypia in their pathological reports, where MIB-1 labeling were 5.9 in the first case and $6.7 \%$ in the second case.

TABLE 1

Demographic and treatment data

\begin{tabular}{|c|c|c|c|}
\hline & whole study & steady after primary treatment & Recurrent or progressive tumor \\
\hline All Patients & 14 & $8(57 \%)$ & $6(43 \%)$ \\
\hline \multicolumn{4}{|l|}{ Age at Diagnosis $(n=14)$} \\
\hline Mean (range) & $28.3(16.2-58.4)$ & $29.2(20.4-54.1)$ & 28.6(16.2-58.4) \\
\hline \multicolumn{4}{|l|}{ Gender $(n=14)$} \\
\hline Male & $8(57 \%)$ & 5 & 3 \\
\hline Female & $6(43 \%)$ & 3 & 3 \\
\hline \multicolumn{4}{|c|}{ Size on MRI (Maximum measurement in $\mathrm{cm}$ ) } \\
\hline Mean (range) & $6.7(3.8-9.6)$ & $6.1(4.8-8.6)$ & $6.8(6.5-9.1)$ \\
\hline Median & 6.1 & 6.1 & 6.7 \\
\hline \multicolumn{4}{|c|}{ Extent of Resection \pm post-operative dose of radiotherapy $(n=14)$} \\
\hline GTR (all) & 5 & 3 & 2 \\
\hline GTR + dose of $R T$ & 1 & 1 & 0 \\
\hline GTR - dose of RT & 4 & 2 & 2 \\
\hline STR (all) & 9 & 5 & 4 \\
\hline STR+ dose of RT & 2 & 1 & 1 \\
\hline STR - dose of RT & 7 & 4 & 3 \\
\hline \multicolumn{4}{|c|}{ MIB-1 tagging index of Tumor (percentage) $(n=12)$} \\
\hline Mean (range) & $3.7(.65-7.7)$ & $2.9(2.0-6.0)$ & $4.8(0.75-7.8)$ \\
\hline Median & 3.4 & 2.3 & 4.8 \\
\hline Required VP shunting & $5(41 \%)$ & 2 & 3 \\
\hline
\end{tabular}


TABLE 2

Progression free survival rates (PFS) in relation to clinical factors

\begin{tabular}{|c|c|c|c|c|}
\hline & $N$ & 2-year PFS & 5-year PFS & p value \\
\hline Total study & 14 & $71 \%(52-87 \%)$ & $52 \%(32-71 \%)$ & \\
\hline \multicolumn{5}{|c|}{ MIB Labelling index } \\
\hline$M I B-1 \leq 4 \%$ & 7 & $89 \%(46-98 \%)$ & $89 \%(46-98 \%)$ & 0.0025 \\
\hline$M I B-1>4 \%$ & 5 & $49 \%(15-75 \%)$ & $11 \%(2-40 \%)$ & \\
\hline \multicolumn{5}{|l|}{ Tumor size } \\
\hline$\leq 6.1$ & 7 & $72 \%(45-86 \%)$ & $51 \%(25-74 \%)$ & 0.79 \\
\hline$>6.1$ & 6 & $76 \%(43-89 \%)$ & $59 \%(22-81 \%)$ & \\
\hline \multicolumn{5}{|c|}{ Overall resection extent } \\
\hline GTR & 5 & $73 \%(36-95 \%)$ & $39 \%(7-72 \%)$ & 0.54 \\
\hline STR & 9 & $75 \%(48-85 \%)$ & $59 \%(36-80 \%)$ & \\
\hline \multicolumn{5}{|c|}{ Resection extent \pm adjuvant radiotherapy } \\
\hline$S T R+R T$ & 2 & $100 \%$ & $65 \%(13-94 \%)$ & .047 \\
\hline$S T R-R T$ & 7 & $71 \%(37-85 \%)$ & $53 \%(24-78 \%)$ & \\
\hline
\end{tabular}

\section{Management of cases with progressive course}

Quite a few executive approaches were applied for the 6 patients who had $\mathrm{CN}$ with progressive course or recurrence. Two patients (30\%) administrated course of chemotherapy. First case who was a course of CCNU had good radiographic images and stable disease for 2 years later. The other one was treated with temozolomide, and had almost three years of stable tumor course before restrained progress. Two other cases (30 \%) had radiosurgery courses. Both showed radiographic images proved the effectiveness of radiation in control of the tumours development on follow ups. The last two patients (33\%) had repeat surgery. One of them was transferred to urgent surgery as a sequelae of intracerebral hemorrhages. The surgery did not improve the neurological condition of the patient and the patient died few days after.

\section{Case illustration}

First case 29 years old lady complained from manifestation of intracranial hypertension in the form of sever persistent headache not responding to medical treatment with blurring of vision frequent vomiting, Tinnitus and urination disorder. Imaging studies of the brain:

* C.T. (Figure 1) that show a large lesion measuring $9 \times 7 \times 5 \mathrm{~cm}$ in both lateral ventricle \& centered on septum pellucidum. There are numerous high density foci concomitant with tumor calcification

* MRI (Figure 2, Figure 3) revealed large minimal enhancing intraventricular mass which has a "soap bubble" appearance on 
T2. It has also broad attachment to the septum pellucidum

* Digital Subtraction Angiography "DSA" (Figure 4) show tumor feeder from Anterior \& posterior choroidal arteries

* 2-deoxy-2[F-18] fluoro-D-glucose positron emission tomography "FDGPET" (Figure 5) show slight accumulation in the left side part of the mass $(60 \%)$

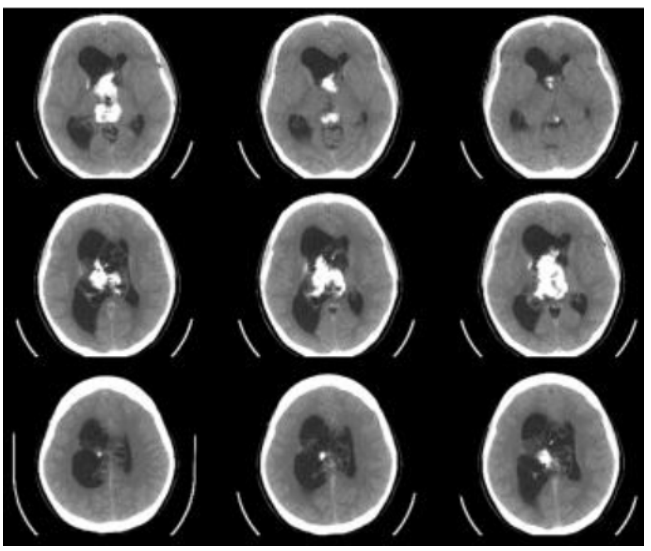

Figure 1 - a Preoperative axial CT images demonstrating a large lesion measuring $9 \times 7 X 5 \mathrm{~cm}$ in both lateral ventricle \& centered on septum pellucidum

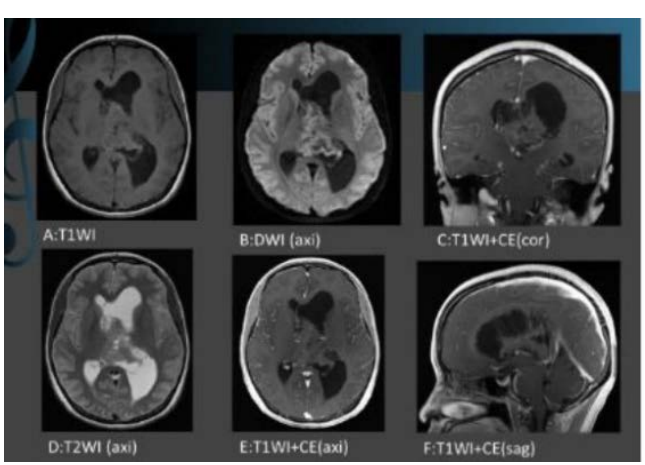

Figure 2 - MRI of the same tumor: A A:T1WI, B:DWI (axi), C:T1WI+CE(cor), D:T2WI (axi), $\mathrm{E}: \mathrm{T} 1 \mathrm{WI}+\mathrm{CE}(\mathrm{axi}), \mathrm{F}: \mathrm{T} 1 \mathrm{WI}+\mathrm{CE}(\mathrm{sag})$

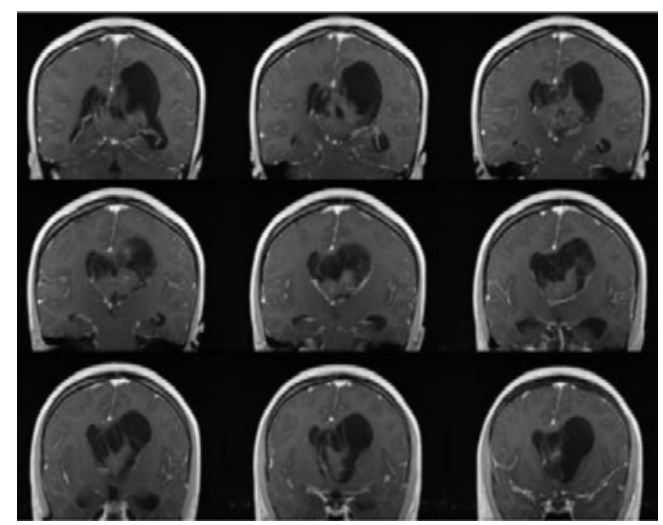

Figure 3 - MRI of the same tumor coronal cuts with contrast show the wide connection to septum pellucidum

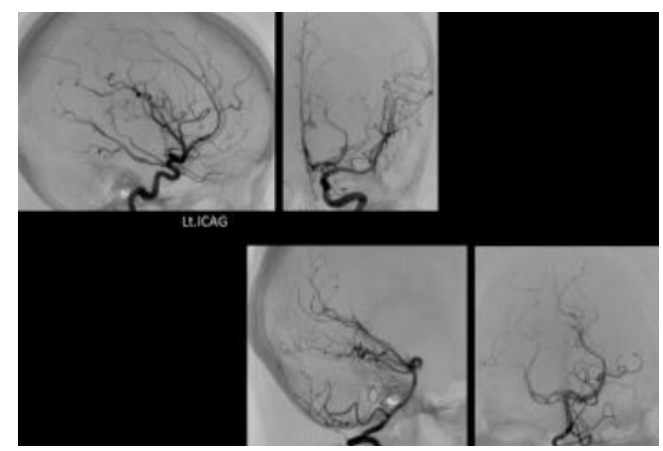

Figure 4 - Digital Subtraction Angiography DSA show feeder from Anterior \& posterior choroidal arteries

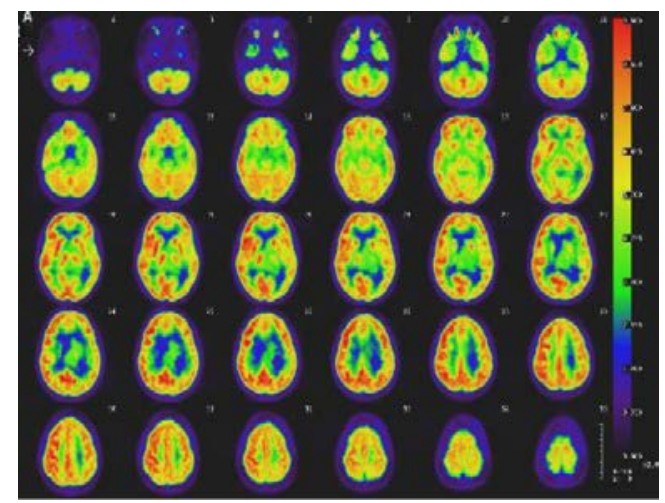

Figure 5 - 2-deoxy-2[F-18] fluoro-D-glucose positron emission tomography FDG-PET show slight accumulation in the left side part of the mass (60\%) 
First step was forming Extra Ventricular drainage "EVD" through frontal burr whole as the patient showed deterioration in conscious level GCS was 11/15. The opening pressure was $40 \mathrm{~cm} \mathrm{H2O}$ (Figures 6, 7).

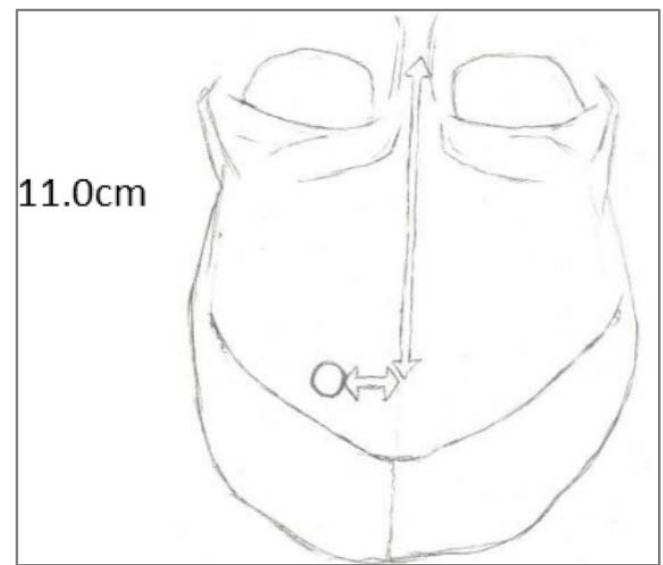

Figure 6 - a diagram for the site of frontal burr hole for EVD

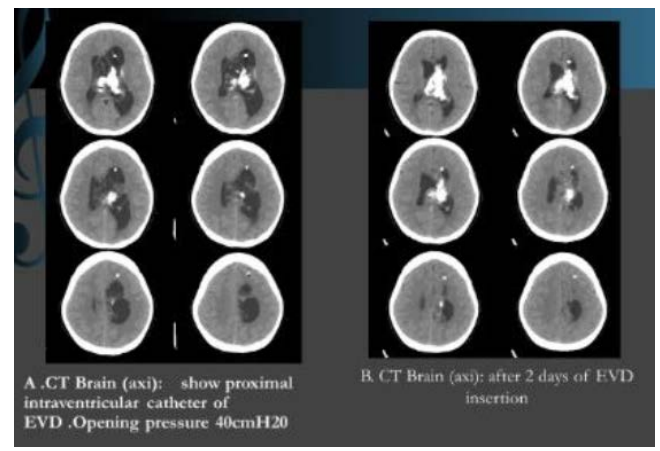

Figure 7 - A.CT Brain (axi): show proximal intraventricular catheter of EVD. Opening pressure 40cmH20. B. CT Brain (axi): after 2 days of EVD insertion

\section{Surgical procedure detail}

- Transcortical trans ventricular approach through middle frontal gyrous. (Figure 8B)

- The tumor outside is a dark purple soft tumor, the inside is a grayish hard tumor with calcification. Tumor removed piece meal Identification of Anterior septal vein \& thalamostriate vein of right lateral ventricle (Figure $8 \mathrm{C}$ )

- Identification of the vascular structures in the field: Anterior septal vein, thalamostriate vein \& Internal cerebral vein of right lateral ventricle at the end stage of tumor resection (Figure 8D,E).

- Open foramen of Monro

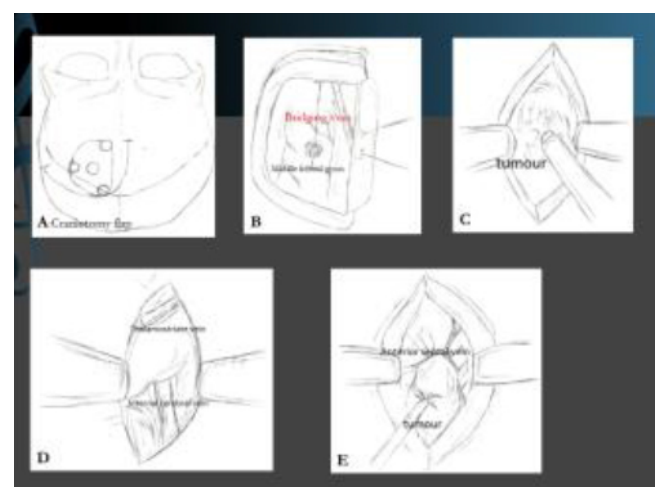

Figure 8 - A-E show diagrams of surgical steps in resection of $\mathrm{CN}$ mass of 29 years old female diagram for the site of frontal burr hole for EVD

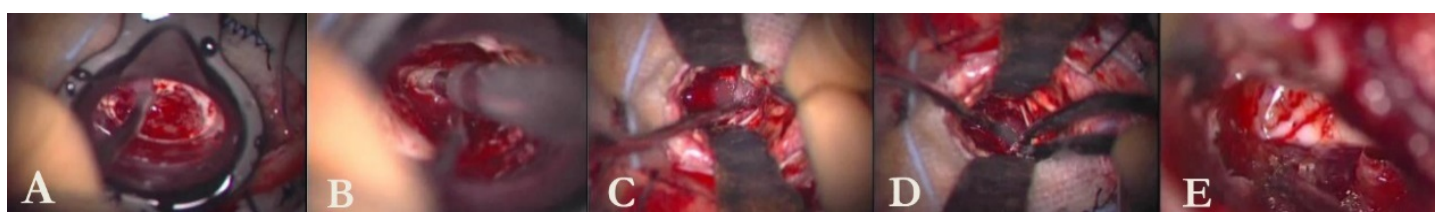

Figure 9 - A-E show microscopic pictures of surgical steps in resection of CN mass of 29 years old female till complete removal 


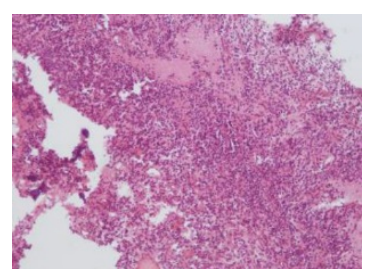

A. HE stain $(\times 100)$

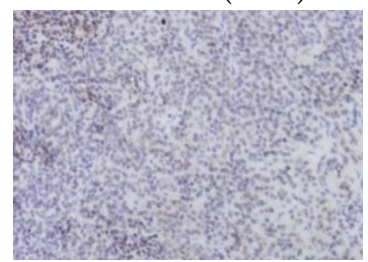

E. Synaptophysin

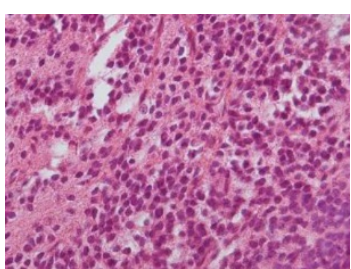

B. HE stain $(\times 400)$

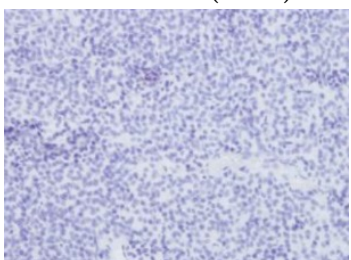

F. NF (-)

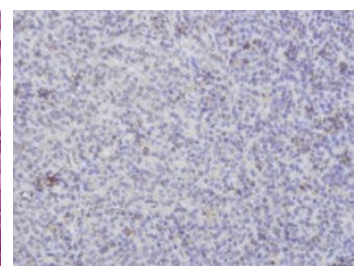

C. GFAP $(+)$

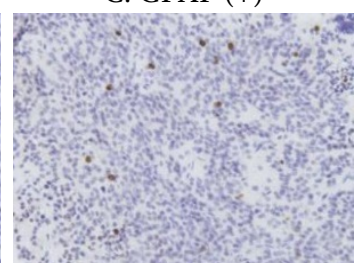

G. MIB - $1(+) 3 \%$

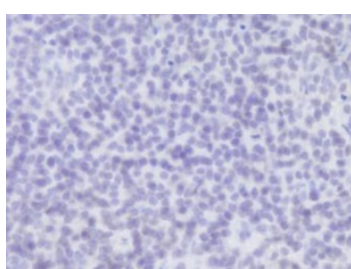

D. Olig2 (-)

Figures 10 - A-G a Microscopic histopathological examination pictures showed: A\&B revealed neurocytic tumor composed of cells with round to oval nuclei [HE stain $(\times 100), 8$ HE stain $(\times 400)$ ]. C. showed positive result for GFAP. D. showed negative result for Oligo2. E. revealed Diffuse immunolabeling for synaptophysin. F. showed negative presence of NF. G The MIB-1 labelling index was about 3\% overall

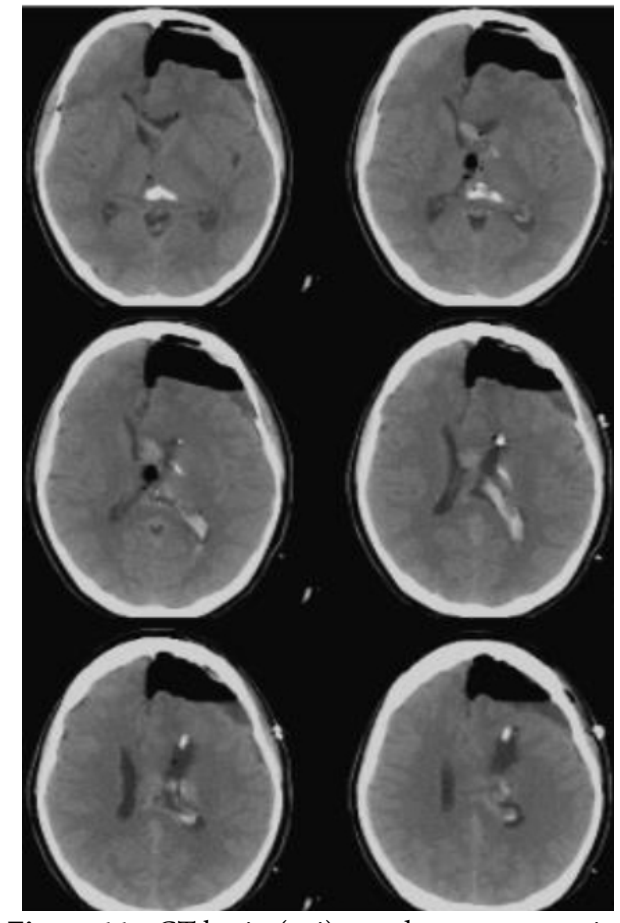

Figure 11 - CT brain (axi) one day post-operative showed complete removal of $\mathrm{CN}$ tumor \& some Pneomocephalous

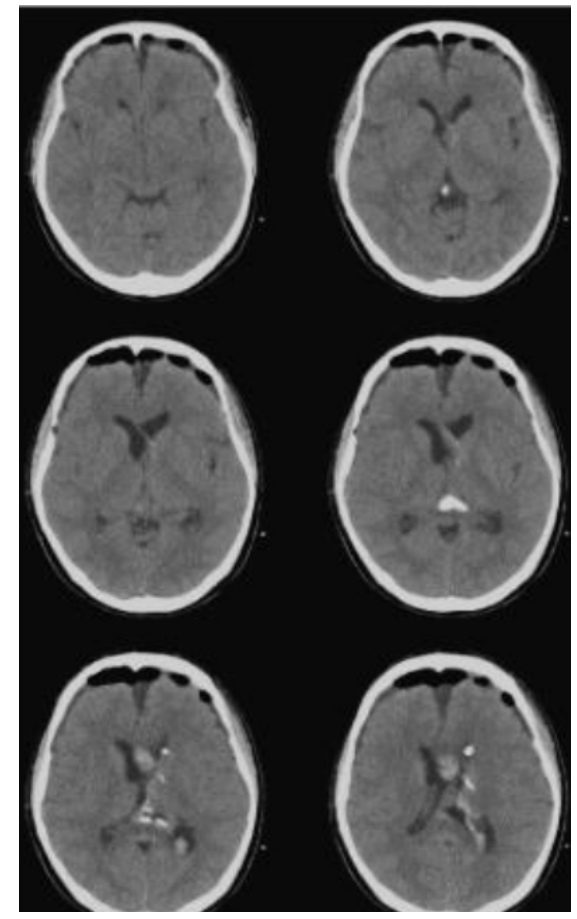

Figure 12 - CT brain (axi) five day post-operative showed complete removal of CN tumor \& Pneomocephalous cessation 


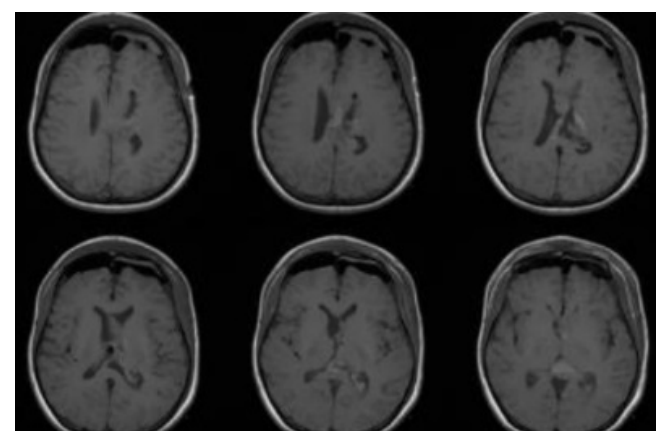

Figure 13 - MRI brain T1WI (axi) five day postoperative showed complete removal of $\mathrm{CN}$ tumor

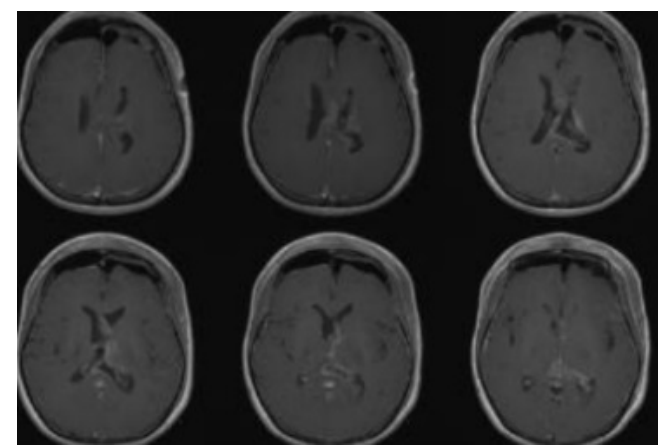

Figure 14 - MRI brain T1WI+CE (axi) five day postoperative showed complete removal of $\mathrm{CN}$ tumor

\section{Discussion}

It has been thought- long time ago -that the worldwide guidelines for management of $\mathrm{CN}$ has not been recognized and remain indistinct, particularly that the effectiveness of RT after tumor surgical removal stays debatable,and this idea has been augmented because of limited cases.In our study, likewise most institutions, we recommend surgical excision as first line therapy [19]. Although the majority of patients will have good prognosis; apparent hazard factors that responsible for fair outcome are growing. GTR had better results than STR, atypical pathological $\mathrm{CN}$ have worse local control OS in comparison to the tumours with classical pathological features, and this has been demonstrated in a collective data of more than 400 patients from international researches [19]. It is currently well recognized that more aggressive behavior is coupled with a higher MIB-1 labeling index $[4,21]$. In our study, only twelve cases had pathological smears for MIB-1 immunohistochemical stain (range $=0.65-7,7$ $\%)$. The recurrent cases possessed a higher mean MIB-1 index. Future treatment guidelines might integrate MIB-1 results as one of other factors that will determine further need of adjuvant treatment subsequent to STR [14].nevertheless, broadly "atypical neurocytoma" as a pathological classification term with high MIB-1 values as a sign for more worse prognosis stay in doubt. Many researches failed to proof any association between high MIB-1 value and possibility of recurrence. Although in this series, even MIBlindex value of more than $2 \%$ was not linked to worse prognosis [1].

One of the early studies to identify the relationship between MIB-1 tagging index and recurrent possibility assumed higher than $2 \%$ as an indicator of more aggressive disease course [14, 21]. A higher threshold has been anticipated based on A meta-analysis achieved on some scientific papers about a decade ago, signifying that $>3 \%$ is interrelated with superior recurrence rates and lower OS [18]. In the current study, notably elevated risk of progression were found in tumors with preliminary MIB-1 indices higher than $4 \%$. This finding make parallel with other studies that suggest higher thresholds for definition of 
atypical pathological features with subsequent more aggressive disease course [9].

On the other hand many researches failed to proof any association between high MIB-1 value and possibility of recurrence. Although in this series, even MIB-lindex value of more than $2 \%$ was not linked to worse prognosis [1].

In spite of the threshold, agreement is present that multimodality treatment must be chosen for managing those tumors with elevated MIB-1 values. The tumours with large volumes that expanded aggressively in lateral ventricle alone or/and the third ventricle, possibly will call for more than single operation using anterior or/and posterior approaches, as in most scientific papers, the neurosurgeons could not accomplish GTR for $\mathrm{CN}$ patients.This fact denoting the technical difficulties in surgical removing of the intraventricular tumors like CN. However, in this study, the degrees of primary surgical excision and lesser primary tumor volume did not look to illustrate a relationship with better local control. Whereas most previous case series $[7,19,22]$ stress that partial resection of $\mathrm{CN}$ tumours linked to high rate of recurrence, but this fact is still debatable. A modern series of 45 central neurocytoma patients data was collected retrospectively, found that a favorable patients outcome beside tumor control was in patients with lesser mitotic values. Meanwhile it did not proof any noteworthy distinction in tumor local control or patient's outcome between GTR and STR [12].

In a new methodical review, resection degree was not associated with better local control [5]. It should be mentioned here that assessment of patients' survival \& quality of life variances between STR and GTR is not feasible, owing to the relatively limited patient's number in this study. The role of post-operative radiation dose in improving tumor local control \& OS was mentioned progressively in many current studies [2].

Considering the intrinsic restriction of limited cases, our figures advocate more favorable tumor local control for patient who had been exposed to post-operative poster dose of radiotherapy in comparison to those patients who did not. The suitable and effective management for patients who had recurrent $\mathrm{CN}$ tumours still uncertain, with many alternatives which may be only single line of treatment or combined to be more than one. This include re-surgery, single or multiple sessions of EBRT, gamma knife (SRS) beside single or multiple cycles of chemotherapy [3]. Similarly, on looking to the current study, the management multimodalities highlights the diversity of these tumours treatment guidelines. Genc et al. have examined employment of SRS for patients with remaining or relapsed $\mathrm{CN}$ tumours in one of the largest series and after median period of follow up approximately 37 months, they reported reduction in tumours volume for $15 / 22$ patients while the $\mathrm{CN}$ tumours size remain stable in $6 / 22$ and only a case showed disease progression [6]. For lesions with lower MIB-1, they described a tendency towards a better response; nevertheless, these findings remain trivial beside its inherited defect of short follow up periods. It was recently found that for recurrent or residual tumors fractionated EBRT and SRS both represent a 
sound option and this was based on a systematic review that also discovered a trivial statistical effect of SRS on enhancement of CN residual tumor local control and improvement of patients' outcomes beside inferior complications rates [5]. The researchers warn that the current evidence is typically low-level. This may advocate that the cons and pros of both treatment lines maintain to be weighed for every patient individually, consequently prospective data would be so helpful. We are in agreement that the maximal harmless $\mathrm{CN}$ tumours microsurgical excision still the golden standard treatment option. The presence of tumor atypia features and high MIB-1 label in pathological examination \& immunohistochemical staining films should guide us to give these patient post-operative dose of radiation. In more detail, the usage of a MIB-1 label value of $4 \%$ as a landmark for tumours atypia and disease aggressiveness is supported by our study findings and we advise co-specialties of concern consultations for those patients. On reviewing our study we can conclude that the post-operative EBRT could diminish the chances of growth for partly surgical removed CN tumours. Lastly, SRS as a modern treatment modality can be a valuable option, especially in recurrent cases.

\section{Conclusion}

The extent of surgical resection may improve the neurological condition but not the survival, Atypia was the most important factor determine the recurrence \& survival while radiotherapy improve the survival quietly.

\section{References}

1. Bertalanffy A, Roessler K, Koperek O, Gelpi E, Prayer D, Knosp E (2005) Recurrent central neurocytomas. Cancer 104:135-142

2. Chen Y-D, Li W-B, Feng J, Qiu X-G (2014) Long-term outcomes of adjuvant radiotherapy after surgical resection of central neurocytoma. Radiat Oncol Lond Engl 9:242

3. Choudhari KA, Kaliaperumal C, Jain A, Sarkar C, Soo MYS, Rades D, Singh J (2009) Central neurocytoma: a multi-disciplinary review. Br J Neurosurg 23:585-595

4. Christov C, Adle-Biassette H, Le Guerinel C (1999) Recurrent central neurocytoma with marked increase in MIB-1 labelling index. Br J Neurosurg 13:496-499,

5. Garcia RM, Ivan ME, Oh T, Barani I, Parsa AT (2014) Intraventricular neurocytomas: a systematic review of stereotactic radiosurgery and fractionated conventional radiotherapy for residual or recurrent tumors. Clin Neurol Neurosurg 117:55-64

6. Genc A, Bozkurt SU, Karabagli P, Seker A, Bayri Y, Konya D, Kilic T (2011) Gamma knife radiosurgery for cranial neurocytomas. J Neurooncol 105:647-657

7. Hallock A, Hamilton B, Ang LC, Tay KY, Meygesi JF, Fisher BJ, Watling CJ, Macdonald DR, Bauman GS (2011) Neurocytomas: long-term experience of a single institution. Neuro-Oncol 13:943-949

8. Hassoun J, Gambarelli D, Grisoli F, Pellet W, Salamon G, Pellissier JF, Toga M. (1982): Central neurocytoma. An electron-microscopic study of two cases. Acta Neuropathol (Berl). 56: 151-6.

9. Kaur G, Kane AJ, Sughrue ME, Oh M, Safaee M, Sun M, Tihan T, McDermott MW, Berger MS, Parsa AT (2013) MIB-1 labeling index predicts recurrence in intraventricular central neurocytomas. J Clin Neurosci Off J Neurosurg Soc Australas 20:89-93

10. Kim C-Y, Kim DG, Joo J-D, Kim YH.( 2015):Clinical outcome and quality of life after treatment of patients with central neurocytoma. Neurosurg Clin N Am.; 26: 83-90. doi: 10.1016/j.nec.2014.09.007

11. Kim JW, Kim DG, Kim IK, Kim YH, Choi SH, Han JH, Park C-K, Chung H-T, Park S-H, Paek SH, Jung HW. (2013): Central neurocytoma: long-term outcomes of multimodal treatments and management strategies based on 30 years' experience in a single institute. Neurosurgery. 72: 407-413-414. doi: 10.1227/NEU.0b013e3182804662 
12. Leenstra JL, Rodriguez FJ, Frechette CM et al (2007) Central neurocytoma: management recommendations based on a 35-year experience. Int J Radiat Oncol Biol Phys 67:1145-1154

13. Louis DN, Ohgaki H, Wiestler OD, et al. (2007), The 2007 WHO classification of tumours of the central nervous system. Acta Neuropathol. 114:97-109.

14. Mackenzie IR (1999) Central neurocytoma: histologic atypia, proliferation potential, and clinical outcome. Cancer 85:1606-1610,

15. Maiuri F, Spaziante R, De Caro ML, Cappabianca P, Giamundo A, Iaconetta G. (1995) Central neurocytoma: clinico-pathological study of 5 cases and review of the literature. Clin Neurol Neurosurg.; 97: 219-28 MIB1.

OMIM.URL: http://www.ncbi.nlm.nih.gov/entrez/dispomim.cgi?id=6 08677. Accessed on: April 3, 2015

17. Paek SH, Han JH, Kim JW, Park CK, Jung HW, Park SH, Kim IH, Kim DG (2008): Long-term outcome of conventional radiation therapy for central neurocytoma. J Neurooncol, 90(1):25-30

18. Rades D, Fehlauer F, Schild SE (2004) Treatment of atypical neurocytomas. Cancer 100:814-817

19. Rades D, Schild SE (2006) Treatment recommendations for the various subgroups of neurocytomas. J Neurooncol 77:305-309
20. Rades D, Schild SE, Fehlauer F (2004) Prognostic value of the MIB-1 labeling index for central neurocytomas. Neurology 62:987-989

21. So“ylemezoglu F, Scheithauer BW, Esteve J, Kleihues P (1997) Atypical central neurocytoma. J Neuropathol Exp Neurol 56:551-556

22. Vasiljevic A, Franc, ois P, Loundou A, Fe'vreMontange M, Jouvet A, Roche P-H, Figarella-Branger D (2012) Prognostic factors in central neurocytomas: a multicenter study of 71 cases. Am J Surg Pathol 36:220227

23. Yang I, Ung N, Chung LK, Nagasawa DT, Thill K, Park J, and Tenn S. (2015) Clinical manifestations of central neurocytoma. Neurosurg Clin N Am.; 26: 5-10. doi: 10.1016/j. nec.2014.09.011

24. Yasargil MG, von Ammon $K$, von Deimling $A$, Valavanis A, Wichmann W, Wiestler OD. (1992) Central neurocytoma: histopathological variants and therapeutic approaches. J Neurosurg. 76: 32-7. doi: 10.3171/ jns.1992.76.1.0032

25. Zhang B, Luo B, Zhang Z, Sun G, Wen J.( 2004) Central neurocytoma: a clinicopathological and neuroradiological study. Neuroradiology. 46: 888-95. doi: 10.1007/ s00234-004-1289-9 